\title{
An Efficient Architecture for Resource Provisioning in Fog Computing
}

\author{
Prof. Minaz Mulla1, Malanbi Satabache², Netravati Purohit ${ }^{3}$
}

\begin{abstract}
${ }^{1}$ Dept of Computer Science \& Engineering, Secab Institute of Engineering \& Technology, Vijaypur, Nauraspur Road Jalnagar Vijaypur ${ }^{2}$ Dept of Computer Science \& Engineering, Secab Institute of Engineering \& Technology, Vijaypur, Nauraspur Road Jalnagar Vijaypur

${ }^{3}$ Dept of Computer Science \& Engineering, Malik sandal Polytechnic, Vijaypur, Nauraspur Road Jalnagar Vijaypur
\end{abstract}

\begin{abstract}
Cloud computing is a model of sharing computing resources over any communication network by using virtualization. Virtualization allows a server to be sliced in virtual machines. Each virtual machine has its own operating system/applications that rapidly adjust resource allocation. Cloud computing offers many benefits, one of them is elastic resource allocation. To fulfill the requirements of clients, cloud environment should be flexible in nature and can be achieve by efficient resource allocation. Resource allocation is the process of assigning available resources to clients over the internet and plays vital role in Infrastructure-as-a-Service (IaaS) model of cloud computing. Elastic resource allocation is required to optimize the allocation of resources, minimizing the response time and maximizing the throughput to improve the performance of cloud computing. Sufficient solutions have been proposed for cloud computing to improve the performance but for fog computing still efficient solution have to be found. Fog computing is the virtualized intermediate layer between clients and cloud. It is a highly virtualized technology which is similar to cloud and provide data, computation, storage, and networking services between end users and cloud servers. This paper presents an efficient architecture for resources provisioning in fog computing environment by using virtualization technique.
\end{abstract}

Keywords: Cloud Computing, Fog Computing, Resource Allocation, virtual machine and virtualization.

\section{Introduction}

Cloud computing is a model of sharing computing resources over any communication network by using virtualization. Virtualization allows a server to be sliced in virtual machines. Each virtual machine has its own operating system/applications that rapidly adjust resource allocation.

\subsection{Cloud computing}

Cloud computing is an emerging technology that can be defined as a tool which provides enormous benefits to their end users. It is an on-demand service model which is remotely available to users, highly scalable and allocates resources to the users in pay as-you-go manner. Cloud computing offers various services as on-demand self service, broad network access, resource pooling, rapid elasticity and measured service. Cloud computing consists of four deployment models. 1). Public cloud 2). Private cloud 3). Hybrid cloud 4). Community cloud [1]. Public cloud provides services for general users over the internet. Private cloud is governed by some private organization for their private use. Hybrid cloud is union of public cloud and private clouds. Community cloud is a kind of private cloud which is shared by some organization having same requirements and objectives.

Cloud computing offers service models are Software-as-aservice (SaaS), Platform-as-a-service (PaaS) and Infrastructure-as-a-service (IaaS) model. The combination of above three models is called XaaS that means anything-as-aService. Through SaaS service cloud offers readymade applications and required software to the end users over the web. PaaS provides platform to make coding or develop own software and application for the end users. IaaS offers virtual hardware and software like computing resource, storage, networks and operating systems to the client machine.

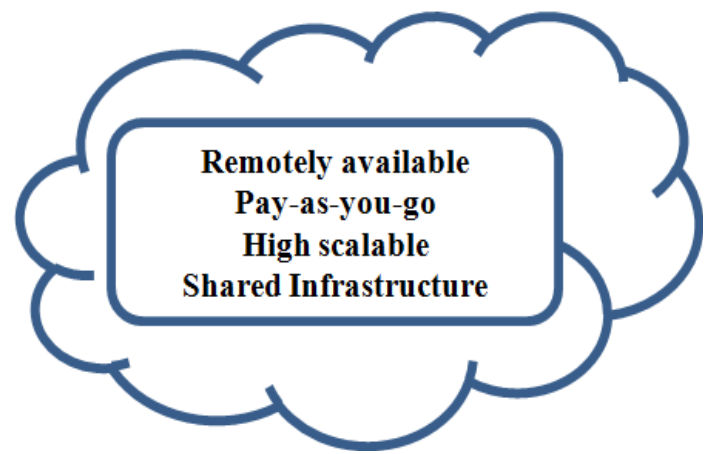

Figure 1.1: Characteristic of Cloud Application

Beyond the cloud computing benefits and applications, it suffers from some issues and inefficiencies. The main characteristics of fog computing is to support applications that require low latency, location awareness, distributed geographical distribution and support for mobility and real time interaction [2]. For optimal utilization of resources, server virtualization is one of the challenging issue in cloud computing.

Server virtualization attempts to increase resource utilization by dividing individual physical server into multiple virtual servers each with its own operation environment and applications through the magic of virtualization [1]. Each virtual server looks and acts just like physical server, multiplying the capacity of any single machine. Figure 1.2, shows the implementation of server virtualization.

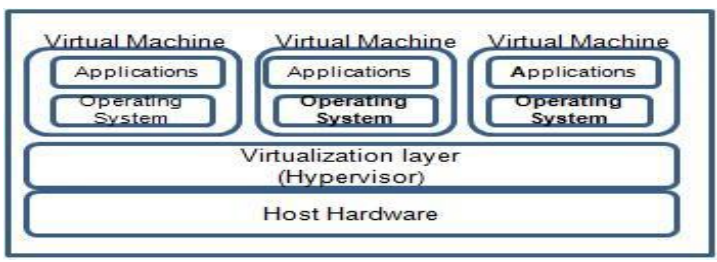

Figure 1.2: Use of Server Virtualization 


\section{International Journal of Science and Research (IJSR) \\ ISSN (Online): 2319-7064}

Index Copernicus Value (2015): 78.96 | Impact Factor (2015): 6.391

Thus proposing an efficient architecture for resource allocation in fog computing environment. Proposed algorithm solves the issues on the requirement of service level elasticity and availability of resources.

\section{Problem Statement}

To resolve the problems of cloud computing, highly virtualized fog computing technology plays major role, which is act between end user and the cloud data centers hosted within the internet.

In the existing resource allocation techniques there is a problem of over-provisioning and under-provisioning; therefore we are going to propose a better architecture in fog environment to overcome this problem.

\section{A Significance of Resource Allocation}

Resource allocation is the systematic approach of allocating available resources to the needed cloud clients over the internet. These resources should be allocated optimally to the applications which are running in the virtualized cloud environment [3]. The order and time of allocation of resources is an important input for an optimal resource allocation. The benefit of resource allocation is that the user does not need to expand on hardware and software system. Server virtualization is used to achieve the user satisfaction and resource utilization ratio in high performance cloud computing. Proposed method provides the even distribution of resources across all the clients in the system .environment to overcome this problem.

\section{System Architecture}

In cloud computing, the efficient resource allocation is the main objective to get the economic benefits. Resource allocation plays an important role to enhance the performance of the entire system and increase the level of customer satisfaction. Server virtualization is an integral part of resource allocation. Server virtualization improves the resource utilization of the system, overall response time and total estimated cost. The work is based on cloud computing technology integrated with fog computing technology. The main features of fog computing is location awareness, mobility, low latency, and distributed geographically. Fog computing is not the replacement of cloud computing, but it reduces the drawbacks of cloud computing and make it efficient. It focuses on the efficient resource allocation algorithm and its applicability in fog environment [6].

Figure 2.1 gives the description of both the computing techniques that how they can be integrated into a single technique. Integration of cloud and fog architecture is difficult to manage because numbers of physical and logical changes are to be made. So, virtualization layer will be required between the cloud layer and fog layer.

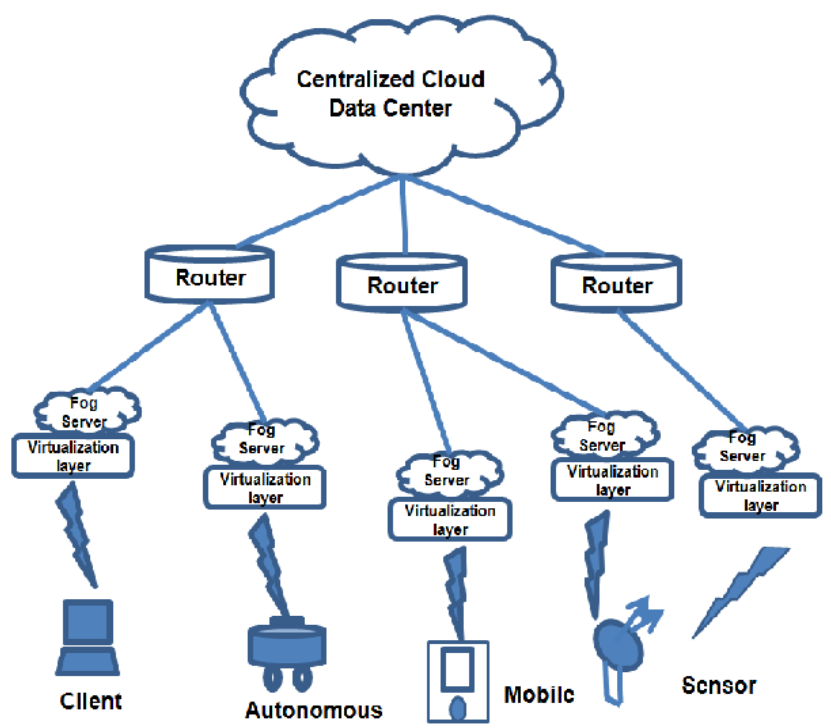

Figure 3.1: Two Layer Architecture for Resource Allocation in Cloud-Fog System

First analyses the various existing algorithms of resource allocation after that we have designed the framework to develop this algorithm. This proposed architecture is implemented for solving the problem related to fault tolerance, resource overflow and underflow as given in figure 3.2

To handle the problem of resource allocation in fog environment, proposed a design model.

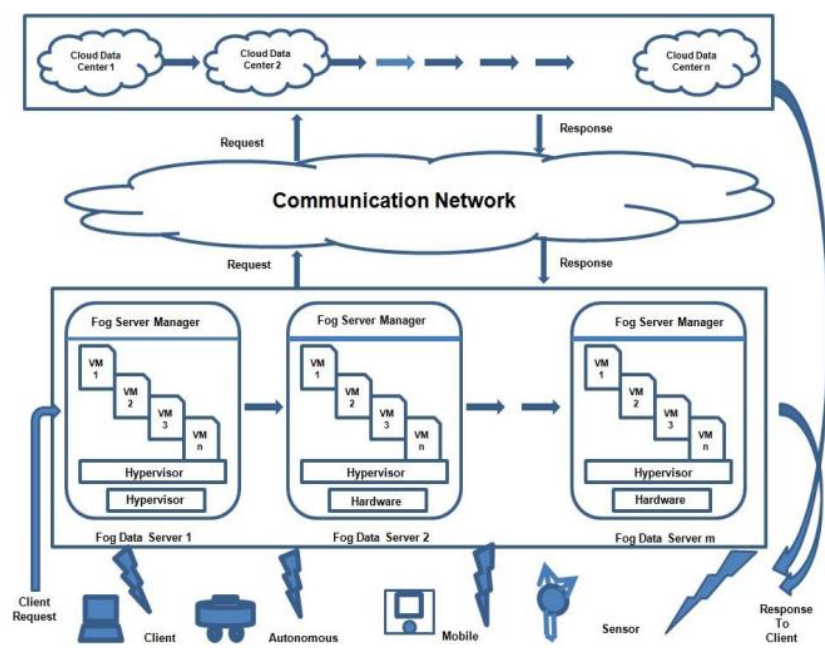

Figure 3.2: Three Layer Architecture for Resource Allocation in Cloud-Fog System

\subsection{Design Model}

This model is designed in a cloud-fog environment. So, the model has three layers named as client layer, fog layer and cloud layer. First, implement the algorithm in client and fog layer to fulfill the requirement of resources for clients. If no resource is available in fog layer than move the request to cloud layer. 


\section{International Journal of Science and Research (IJSR) \\ ISSN (Online): 2319-7064 \\ Index Copernicus Value (2015): 78.96 | Impact Factor (2015): 6.391}

Step 1: All the data centers will be arranged in fog layer and in cloud layer. Each fog layer has number of fog data server (FS) and cloud layer has number of cloud data center (CS).

Step 2: Each fog data server (FS) will contains fog server manager (FSM) which will check the availability of the processor and have the responsibility to manage the VM's.

Step 3: Initially, any client will submit their request to any fog data server (FS), and then fog data server loads the request to its fog server manager (FSM).

Step 4: Fog server manager (FSM) will process the service request in following conditions.

I. If all the requesting processors are available to first fog data server, then it loads the result to the client and client sends an acknowledgement to its fog server manager to update the status of the list.

II. If only some requesting processors are available to fog data server, then total task is divided into number of subtasks as per availability.

III. If fog data server is in allocated but early release state, then client will wait for minimum time constraint; then loads the request to fog data server.

IV. If all processors receive at one fog data server but some are failing during processing, then it will again process the request as in II condition.
V. If no processor is available in fog data servers within its fog cluster, then the request is propagated to cloud data server.

Step 5: If the sender has not received the result of their request within maximum allotted time, then client will wait for processing.

Step 6: For further processing client request is propagated to cloud data server (CS).

Step 7: The cloud data server will provide the processor to client directly to increase the response time and sends an acknowledgement to respective fog server manager.

\section{Result Comparison}

Finally, an analytical comparison is done between various existing algorithms and the proposed efficient resource allocation (ERA) algorithm is given in table 1. It is shown to be clear that our proposed technique is better among existing techniques in terms of response time, bandwidth utilization, less power consumption, reduced data traffic over the internet and low latency. It also remove the problem of server overflow and also found better strategy of resource allocation in fog computing high performance environment.

Table 1: A Comparative Analysis between Existing Algorithms and Proposed Algorithm

\begin{tabular}{|c|c|c|c|c|c|}
\hline \multicolumn{2}{|c|}{ Algorithm\Matrices } & $\begin{array}{c}\text { Three layer } \\
\text { processing }\end{array}$ & $\begin{array}{c}\text { Overall } \\
\text { Maximum } \\
\text { Response Time }\end{array}$ & $\begin{array}{c}\text { Data Center Processing } \\
\text { Time Maximum(ms) }\end{array}$ & $\begin{array}{c}\text { Total Cost } \\
\text { (Virtual Machine } \\
+ \\
\text { Data Transfer(\$)) }\end{array}$ \\
\hline \multirow{2}{*}{$\begin{array}{c}\text { Existing } \\
\text { Algorithms }\end{array}$} & $\begin{array}{c}\text { Reconfigure } \\
\text { Dynamically with } \\
\text { Load balancing }\end{array}$ & Client to cloud & 632.87 & 18.0 & 4.27 \\
\cline { 2 - 6 } & $\begin{array}{c}\text { Optimize Response } \\
\text { time }\end{array}$ & Client to cloud & 630.11 & .71 & .90 \\
\hline \multirow{2}{*}{$\begin{array}{c}\text { Proposed } \\
\text { Algorithm }\end{array}$} & $\begin{array}{c}\text { Efficient resource } \\
\text { allocation(ERA) }\end{array}$ & Client to fog & 63.41 & .11 & 2.59 \\
\cline { 3 - 6 } & Fog to cloud & 246.12 & 1.01 & .57 \\
\hline
\end{tabular}

\subsection{Result Analysis}

Results shown in table, It can compare overall maximum response time, data center processing time maximum and total cost of virtual machine and data transfer cost between existing algorithms and proposed algorithm. Figure 4.1(a), 4.2(b), 4.3(c) shows comparison of the results. Existing algorithms are reconfiguring dynamically with load balancing (RDLB) and optimize response time policy (ORT). The proposed algorithm is efficient resource allocation (ERA). For final result of the proposed algorithm, calculated the result of client to fog layer and then fog to cloud layer. Then the overall result will be the summation of both the results. In our algorithm taking the fog layer as the interface between the client and cloud, so mostly all the requests are fulfill by the fog layer and resources are allocated by the fog data server. But in any case if fog data server is not able to provide the resources, then the fog data server request is forwarded to cloud data center for resource allocation.
Overall Max.Response tim e(m s)

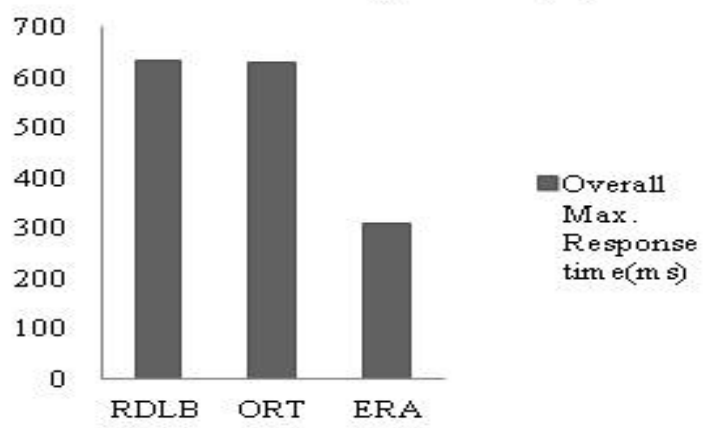

Figure 4.1(a): Comparison of Overall Maximum response time (ms) 


\section{International Journal of Science and Research (IJSR) \\ ISSN (Online): 2319-7064}

Index Copernicus Value (2015): 78.96 | Impact Factor (2015): 6.391

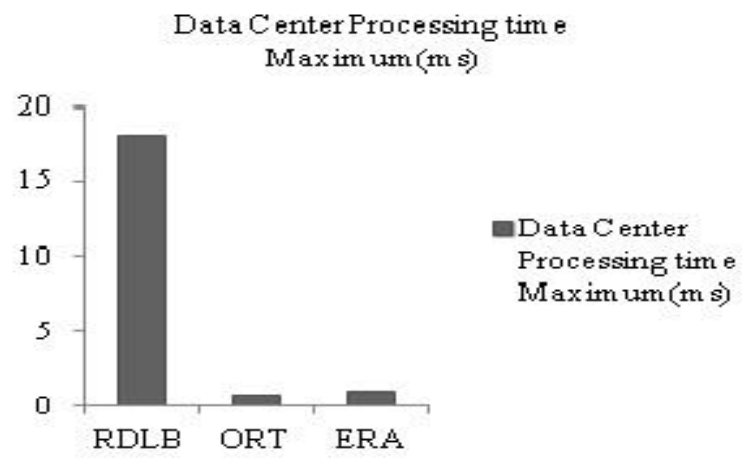

Figure 4.2(b): Comparison of Data Center Processing time Maximum (ms)

TotalCost $(\mathrm{V}$ irtual Machine+Data

Transfer( $(\$))$

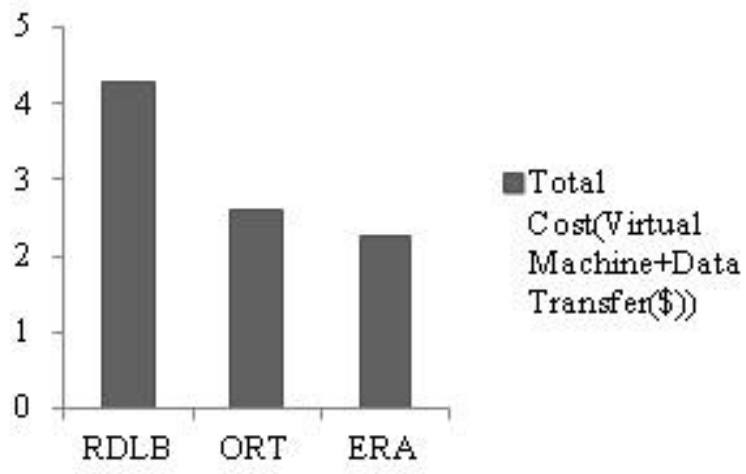

Figure 4.3(c): Comparison of Total Cost (\$)

Figure 4.1(a) shows the comparison of overall response time among the algorithms. Calculated the response time of our proposed algorithm (ERA) is $309.53 \mathrm{~ms}$, it is the summation of two results, ,two results are $63.41 \mathrm{~ms}$ and $246.12 \mathrm{~ms}$. The result for client-fog layer is $63.41 \mathrm{~ms}$ and the result for fogcloud layer is $246.12 \mathrm{~ms}$. The response time of reconfigure dynamically with load balancing (RDLB) is $632.87 \mathrm{~ms}$ and the overall response time of optimize response time is 630.11 ms. It can easily determine that the response time of our proposed algorithm is very less, so if client request for processors to fog data server then fog data server allocate that resource to the client immediately.

Figure 4.2(b) shows the comparison of data center processing time of different existing algorithms and proposed algorithm. It can see from the table 1 that the data center processing time for client-fog layer is .90 ms and for fog-cloud layer is $.11 \mathrm{~ms}$. So total data center processing time for our proposed algorithm is $1.01 \mathrm{~ms}$. The data center processing time for reconfigure dynamically with load balancing (RDLB) and optimize response time (ORT) algorithm is $18.0 \mathrm{~ms}$ and .71 ms respectively. From the graph we can easily analyses that in comparison to RDLB algorithm our proposed algorithm is far better and in comparison to ORT algorithm, our algorithm is just about equal.

In figure 4.3(c), our proposed algorithm (ERA) gives the minimum total cost in comparison to both existing algorithms. The total cost is obtained by the cost of virtual machines and data transfer cost used in the system. The total cost of client-fog layer is $1.70 \$$ and the fog-cloud layer total cost is .57 \$. So the total of our proposed algorithm (ERA) is 2.27 \$. From the table 1, we can determine that the total cost of reconfigure dynamically with load balancing (RDLB) and optimize response time (ORT) algorithm is 4.27 \$ and 2.59 \$ respectively.

From this data analysis, proposed algorithm can effectively minimize the overall response time, data center processing time and total cost. So, proposed strategy fully utilizes the bandwidth of the system, provide effective response time, data processing time and reduce data traffic over the internet in fog computing environment to allocate the resources. So it can define that proposed algorithm fulfills the requirement of resources to the clients immediately which is the objective of our algorithm or resources are provisioned optimally to the applications which are running in the virtualized cloud environment.

Now, it can easily find that proposed algorithm can effectively minimize the overall response time, data center processing time and total cost. So, proposed strategy fully utilizes the bandwidth of the system, provide effective response time, data processing time and reduce data traffic over the internet in fog computing environment to allocate the resources.

\section{Conclusion}

Fog computing is used in work because it improve efficiency of cloud computing and reduce the amount of data that needs to be transported to the cloud for data processing and storage. It is inefficient to transmit all the data of Internet of Thing (IoT) and sensors to the cloud, fog computing deal with this problem. So fog is meant to deliver the idea that the benefits of cloud computing can be brought closer to the client. This gives the idea about the significance of efficient resource allocation and its related concepts. Cloud computing is difficult to understand without resource allocation because it provides reduced infrastructure cost and elastic scalability. It survey various existing algorithms related to optimal resource allocation and different scheduling techniques. This is efficient resource allocation architecture and algorithm (ERA) has been proposed and implemented on cloud analyst tool to test the performance of the proposed technique in the fog environment. By implementing the proposed strategy, It can find that the proposed strategy can be allocated resources in optimized way and better than existing algorithms in terms of overall response time, data transfer cost and bandwidth utilization in fog computing environment. This document shows the comparison of existing resource allocation strategy with the proposed algorithm in terms of overall estimated response time and cost.

\section{References}

[1] Kamyab Khajehei, "Role of virtualization in cloud computing", International Journal of Advance Research in Computer Science and Management Studies Volume 2, Issue 4, April 2014. 
[2] Ivan Stojmenovic, sheng Wen, "The Fog Computing Paradigm: Scenarios and security issues" Proceedings of the IEEE International Fedrerated Conference on Computer Science and Information Systems, 2014, pp. $1-8$.

[3] N.R.RamMohan, E.Baburaj "Resource Allocation Techniques in Cloud computing-Research Challenges for Applications", Proc. Of the IEEE Fourth International Conference on Computational Intelligence and Communication Networks, 2012, pp. 556-560.

[4] Aditya Marphatia, Aditi Muhnot, Tanveer Sachdeva, Esha Shukla, Prof. Lakshmi Kurup," Optimization of FCFS Based Resource Provisioning Algorithm for Cloud Computing" , IOSR Journal of Computer Engineering (IOSR-JCE) e-ISSN: 2278-0661, p- ISSN: 22788727Volume 10, Issue 5 (Mar. - Apr. 2013), PP 01-05.

[5] Swati Agarwal, Shashank Yadav, Arun Kumar Yadav," An architecture for elastic resource allocation in Fog computing", International Journal of Computer Science and Communication, Vol. 6 Number 2 April - Sep 2015 pp. 201-207.

[6] "An Efficient Architecture and Algorithm for Resource Provisioning in Fog Computing" I.J. Information Engineering and Electronic Business, 2016, 1, 48-61 Published Online January 2016 in MECS (http://www.mecs-press.org/) DOI: 10.5815/ijieeb.2016.01.06

[7] Eman Elghoneimy, Othmane Bouhali, Hussein Alnuweiri, "Resource Allocation and scheduling in Cloud Computing", Proc. Of the IEEE International Workshop on Computing, Networking and Communications, 2012, pp. $309-314$.

\section{Author Profile}

Asst. Prof. Minaz Mulla received M-Tech degree from VTU jnan sangam Belgaum. Now I am currently working as an Asst Professor in Computer Science Dept, SIET Vijayapur.

Miss. Malanbi Satabache received the Bachelor of Engineering in Information Science and Engineering from Secab Institute of Engineering \& Technology in the year. Now I am currently pursuing M.-Tech in Computer Network Engineering in Secab institute Of Engineering \& Technology. Vijayapur 